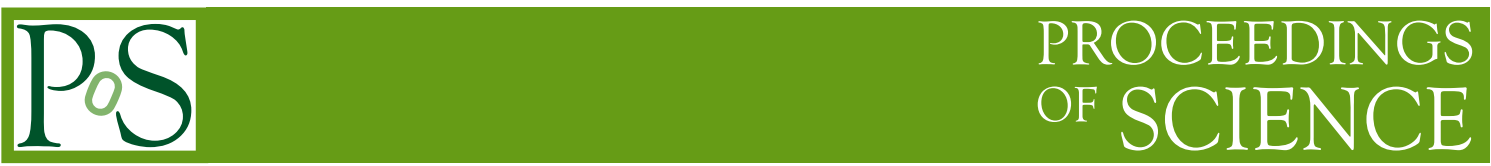

\title{
The ENUBET neutrino beam
}

\section{F. Terranova*}

Phys. Dep. Università di Milano-Bicocca and INFN, Milano, Italy

E-mail: francesco.terranova@cern.ch

F. Acerbi, G. Ballerini, M. Bonesini, C. Brizzolari, G. Brunetti, M. Calviani, S. Carturan, M.G. Catanesi, S. Cecchini, F. Cindolo, G. Collazuol, E. Conti, F. Dal Corso, G. De Rosa, C. Delogu, A. Falcone, B. Goddard, A. Gola, R.A. Intonti, C. Jollet, V. Kain, B. Klicek, Y. Kudenko, M. Laveder, A. Longhin, P.F. Loverre, L. Ludovici, L. Magaletti, G. Mandrioli, A. Margotti, V. Mascagna, N. Mauri, A. Meregaglia, M. Mezzetto, M. Nessi, A. Paoloni, M. Pari, E. Parozzi, L. Pasqualini, G. Paternoster, L. Patrizii, C. Piemonte, M. Pozzato, F. Pupilli, M. Prest, E. Radicioni, C. Riccio, A.C. Ruggeri, G. Sirri, M. Soldani, M. Stipcevic, M. Tenti, F. Terranova, M. Torti, E. Vallazza, F. Velotti, M. Vesco, L. Votano

Fondazione Bruno Kessler (FBK) and INFN TIFPA, Trento, Italy; INFN, Sezione di Milano-Bicocca, Milano, Italy; INFN, Sezione di Bologna, Bologna, Italy; CENBG, Universite de Bordeaux, CNRS/IN2P3, 33175 Gradignan, France; INFN, Laboratori Nazionali di Frascati, Frascati (Rome), Italy; INFN, Sezione di Roma 1, Rome, Italy; Phys. Dep. Università di Bologna, Bologna, Italy; Phys. Dep. Università di Milano-Bicocca, Milano, Italy; INFN Sezione di Trieste, Trieste, Italy; INFN Sezione di Padova, Padova, Italy; Phys. Dep. Università di Padova, Padova, Italy; INFN Sezione di Bari, Bari, Italy; CERN, Geneve, Switzerland; Phys. Dep. Università La Sapienza, Rome, Italy; DISAT, Università degli Studi dell'Insubria, Como, Italy; INFN, Sezione di Napoli, Napoli, Italy; Phys. Dep., Universitá "Federico II" di Napoli, Napoli, Italy; Institute of Nuclear Research of the Russian Academy of Science, Moscow, Russia; Center of Excellence for Advanced Materials and Sensing Devices, Ruder Boskovic Institute, Zagreb, Croatia

ENUBET has been designed to monitor lepton production in the decay tunnel of neutrino beams at single particle level and to provide a $1 \%$ measurement of the neutrino flux at source. In particular, the three body semileptonic decay of kaons monitored by large angle positron production offers a fully controlled $v_{e}$ source at the GeV scale for a new generation of short baseline experiments. The ENUBET Collaboration presented at ICHEP the first end-to-end simulation of the beamline and a review of the performance of this non-conventional technique. Special emphasis has been given to the new static focusing system that was validated in 2018 . We also discussed the performance of the positron tagger tested at CERN in 2017-2018 and the expected sensitivity of ENUBET for $v_{e}$ and $v_{\mu}$ events.

The 39th International Conference on High Energy Physics (ICHEP2018)

4-11 July, 2018

Seoul, Korea

\footnotetext{
*Speaker.
} 


\section{Monitored neutrino beams and the ENUBET project}

The knowledge of the initial flux in conventional neutrino beams represents the main limitation for a precision (1\%) measurement of $v_{e}$ and $v_{\mu}$ cross sections. Such limitation is addressed by the "monitored neutrino beams" [1] and in particular by ENUBET [2]: a facility where the only source of electron neutrino is the three body semileptonic decay of the kaons, $K^{+} \rightarrow \pi^{0} e^{+} v_{e}\left(K_{e 3}\right)$. The ERC ENUBET ("Enhanced NeUtrino BEams from kaon Tagging”) project [3] is aimed at building detectors that identify positrons from $K_{e 3}$ decays while operating in the harsh environment of a neutrino beam decay tunnel. The project addresses all accelerator challenges of monitored neutrino beams: the proton extraction scheme, the focusing and transfer line, the instrumentation of the decay tunnel and the assessment of the physics performance. ENUBET is a proposed narrow band beam with a short $(\sim 20 \mathrm{~m})$ transfer line followed by a $40 \mathrm{~m}$ long decay tunnel. Unlike most of the beams currently in operation, the decay tunnel is not placed in front of the focusing system (horns) and the proton extraction length is slow (a few $\mathrm{ms}$ in the horn option and $2 \mathrm{~s}$ in the static focusing option). Particles produced by the interaction of protons on the target are focused, momentum selected (momentum bite: 10\%) and transported at the entrance of the tunnel. Off-momentum particles are mostly low energy pions, electron, positron, photons from tertiary interactions in the collimators and muons from pion decay crossing the collimators. The rates of these particles in the decay pipe are many orders of magnitude smaller than those of currently operating beams and the production of particles (e.g. positrons from kaons) in the decay tunnel can be monitored at single particle level by instrumenting a fraction of the decay tunnel.

\section{The ENUBET beamline and instrumentation}

Single-particle monitoring is possible only if the duration of the proton extraction is slower than current long-baseline neutrino beams (tens of $\mu \mathrm{s}$ ) in order to keep the particle rate in the decay tunnel well below $1 \mathrm{MHz} / \mathrm{cm}^{2}$. The maximum length of the proton extraction spill depends on the implementation of the focusing system for the secondary particles produced in the target. A hornbased system cannot be sourced in DC mode and the maximum current pulse duration is $\sim 10 \mathrm{~ms}$. On the other hand, a static focusing system can be operated in DC mode and proton extractions up to several seconds can be envisaged. ENUBET is pursuing both options and developing a dedicated proton extraction scheme for ms-long ("burst-mode extraction") and second-long ("slow extraction") extractions. The burst mode consists of extracting many consecutive spills (duration: a few ms) within one macro SPS spill (duration: $\sim 4 \mathrm{~s}$ ). This scheme is compatible with the use of magnetic horns in the ENUBET transfer line. We are currently implementing the extraction scheme in the SPS in a proof-of-concept version and testing it before the LHC Long Shutdown 2 (LS2). The optimization of the machine parameters will be based on the outcome of this measurement campaign and will be validated after LS2.

Primary proton interactions in the target were simulated with FLUKA 2011. The target we considered up to now is a $1 \mathrm{~m}$ long Beryllium cylinder. This target is being replaced by a Graphite target, which eases substantially the thermo-mechanical design and provide similar yields as the Be target and small secondary interaction effects. The results presented at ICHEP are based on 
the CERN-SPS as proton driver (400 GeV protons) but other existing proton drivers have been considered as well (120 GeV and $30 \mathrm{GeV}$ protons).

The beamline optics was optimized using TRANSPORT for a central momentum of $8.5 \mathrm{GeV}$ and a momentum bite of $10 \%$. The best configuration achieved so far consists of a quadrupole triplet followed by a bending dipole and another quadrupole triplet. The dipole and all the quadrupoles have an aperture radius of $15 \mathrm{~cm}$. The dipole field is $1.8 \mathrm{~T}$ providing a bending angle of $7.4^{\circ}$. Fields at pole in the quadrupoles are in the range 5-11 kG. This configuration was implemented in G4Beamline with the addition of absorbers between elements, a Tungsten foil after the target to screen positrons that would otherwise reach the tagger and a low power hadron dump at the end of the decay tunnel.

The particle yields at the entrance of the tunnel are summarized in Tab. 1.

\begin{tabular}{||c|c|c|c|c|c|c||}
\hline Focusing & $\begin{array}{c}\pi^{+} / \text {POT } \\
{\left[10^{-3}\right]}\end{array}$ & $\begin{array}{c}K^{+} / \text {POT } \\
{\left[10^{-3}\right]}\end{array}$ & $\begin{array}{c}\text { Extraction } \\
\text { Length }\end{array}$ & $\begin{array}{c}\pi^{+} / \text {cycle } \\
\left(10^{10}\right)\end{array}$ & $\begin{array}{c}\mathrm{K}^{+} / \text {cycle } \\
\left(10^{10}\right)\end{array}$ & $\begin{array}{c}\text { Factor } \\
\text { w.r.t. [1] }\end{array}$ \\
\hline \hline Horn-based & 77 & 7.9 & $2 \mathrm{~ms}$ & 347 & 36 & $\times 2$ \\
\hline Static & 19 & 1.4 & $2 \mathrm{~s}$ & 86 & 6.3 & $\times 4$ \\
\hline
\end{tabular}

Table 1: Expected rates of $\pi^{+}$and $K^{+}$in $[6.5 \div 10.5 \mathrm{GeV}]$ range at the decay tunnel entrance for the two possible focusing schemes. The improvement factor in kaon transport with respect to Ref. [1] is shown in the last column.

Note in particular that the kaon yield in the static focusing system is four times larger than the original ENUBET proposal [1] due to the re-optimization of the beam optics. On the other hand, the horn-based option still retains the highest yield (useful mesons per proton-on-target).

The ENUBET instrumented decay tunnel consists of a calorimeter for $e^{+} / \pi^{+}$separation and of an inner light-weight detector for $e^{+} / \pi^{0}$ separation and timing (" $t_{0}$-layer"). During 2016-2018 we performed tests in four two-week slots of data taking to validate prototypes for the $t_{0}$-layer and the calorimeters both in shashlik and lateral readout modes $[3,4,5,6]$. At present, both options fulfill the requirements of ENUBET and a final decision will be taken in 2019 based on the result of the tests that will be done at CERN in October 2018.

Particles inside the tunnel are identified by the energy deposition pattern in the calorimeter and photon veto. The positrons are selected with a signal-to-noise ratio of $\sim 0.5$ and an efficiency of $\sim 20 \%$, which is appropriate for the neutrino flux monitoring at the per-cent level. This level of reconstruction is achieved for the static focusing system assuming a complete extraction of the protons from the SPS in a super-cycle $\left(4.5 \times 10^{13}\right.$ pot in a $2 \mathrm{~s}$ slow extraction).

\section{The ENUBET narrow band beam}

Assuming $4.5 \times 10^{19}$ pot at CERN-SPS, about $1.13 \times 10^{6} v_{\mu}^{C C}$ and $1.4 \times 10^{4} v_{e}^{C C}$ interactions will be observed at a neutrino detector (500 t fiducial mass) located $50 \mathrm{~m}$ from the end of the tunnel.

A narrow band beam as ENUBET not only provides a beam with a precisely measured flux but also a measurement of the neutrino energy that does not rely on the reconstruction of final state particles. This feature ("narrow-band off-axis technique") results from the narrow momentum 
bandwidth of the beam and the finite transverse dimension of the neutrino detector. This feature can be exploited for neutrino detectors that have a size comparable or larger than ICARUS at Fermilab or the ProtoDUNE detectors [7] at CERN.

The energy measurement exploits the correlation between the energy of the neutrino interacting in the detector and the radial distance $(R)$ of the interaction vertex from the beam axis. By selecting interactions in radial windows of $\pm 10 \mathrm{~cm}$ at $R$ equal to $0.5 \mathrm{~m}, 1.5 \mathrm{~m}$ and $2.5 \mathrm{~m}$, we collect samples of about $15.7 \times 10^{4}, 6.4 \times 10^{4}$ and $2.9 \times 10^{4} v_{\mu}^{C C}$ events respectively in the energy range of interest for future long baseline oscillation experiments. The incoming neutrino energy can be determined with a precision given by the pion peak width of the spectrum at a fixed $R$. It ranges from $7 \%$ at $3.5 \mathrm{GeV}$ to $22 \%$ at $0.8 \mathrm{GeV}$ as shown in Fig. 1.

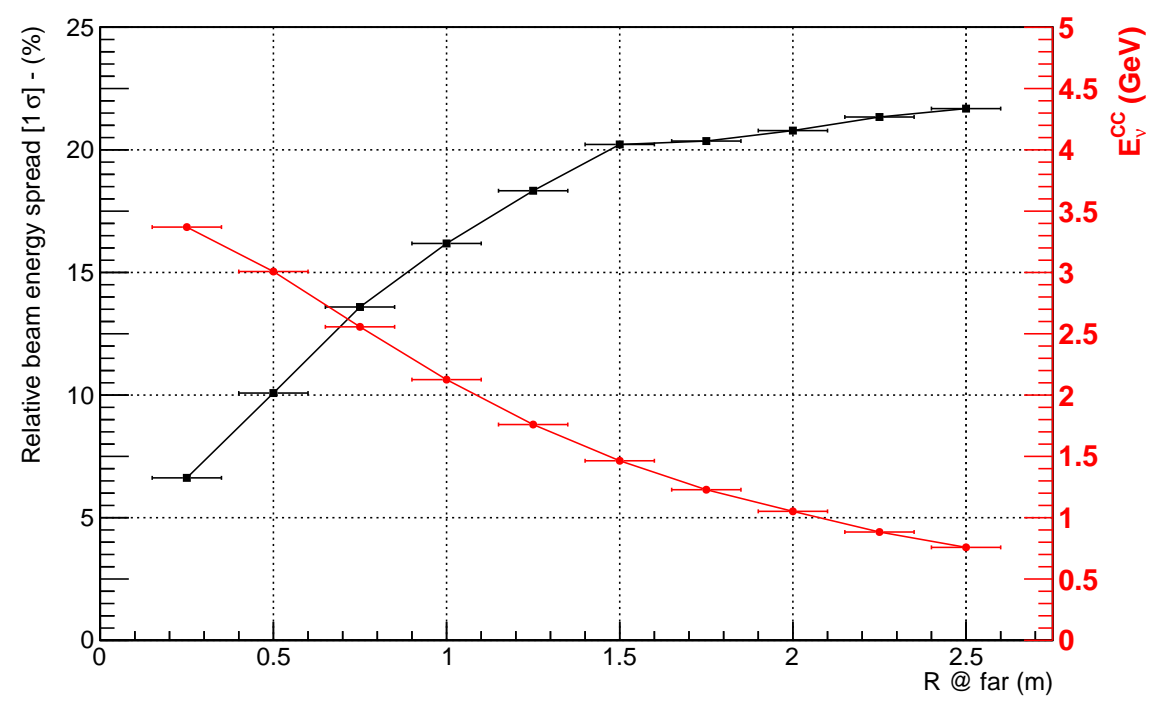

Figure 1: Beam energy spread (in black) and peak energy (in red) as a function of the distance $R$ of the interaction vertex at the detector from the beam axis.

\section{References}

[1] A. Longhin, L. Ludovici and F. Terranova, Eur. Phys. J. C 75 (2015) 155.

[2] A. Berra et al., CERN-SPSC-2016-036, SPSC-EOI-014, Geneva, 2014.

[3] Documentation available at http://enubet.pd.infn.it/ .

[4] A. Berra et al., Nucl. Instrum. Meth. A 830 (2016) 345.

[5] A. Berra et al., IEEE Trans. Nucl. Sci. 64 (2017) 1056.

[6] G. Ballerini et al., JINST 13 (2018) P01028.

[7] See e.g. L. Manenti, arXiv:1705.05669 [physics.ins-det] and references therein. 